\title{
molecules
}

ISSN 1420-3049

Communication

www.mdpi.com/journal/molecules

\section{Ring Cleavage Reactions of Methyl $\alpha$-D-Allopyranoside Derivatives with Phenylboron Dichloride and Triethylsilane}

\author{
Masaru Kojima *, Yutaka Nakamura, Yuusuke Ito and Seiji Takeuchi \\ Niigata University of Pharmacy and Applied Life Sciences, 265-1 Higashijima, Akiha-ku, \\ Niigata 956-8603, Japan \\ * Author to whom correspondence should be addressed; E-Mail: masaru@nupals.ac.jp; \\ Tel.: +81-250-25-5165; Fax: +81-250-25-5021.
}

Received: 31 October 2011; in revised form: 25 November 2011 / Accepted: 5 December 2011 / Published: 13 December 2011

\begin{abstract}
In the course of our studies on the regioselective carbon-oxygen bond cleavage of the benzylidene acetal group of hexopyranosides with a reducing agent, we found that a combination of a Lewis acid and a reducing agent triggered a ring-opening reaction of the pyranose ring of methyl $\alpha$-D-allopyranosides. The formation of an acyclic boronate ester by the attachment of a hydride ion at $\mathrm{C}-1$ indicated that the unexpected endocyclic cleavage of the bond between the anomeric carbon atom and the pyranose ring oxygen atom proceeded via an oxacarbenium ion intermediate produced by the chelation between O5/O6 of the pyranoside and the Lewis acid, followed by nucleophile substitution with a hydride ion at $\mathrm{C} 1$.
\end{abstract}

Keywords: methyl $\alpha$-D-allopyranoside; endocyclic cleavage; phenylboron dichloride; triethylsilane

\section{Introduction}

Lewis-acid-induced regioselective carbon-oxygen bond cleavage of the benzylidene acetal group of hexopyranosides with a reducing agent is an important reaction in carbohydrate chemistry for the syntheses of complex oligosaccharides and glycoconjugates. Until now, various reagent systems [1-12] and investigations of the detailed mechanistic pathway $[13,14]$ have been reported for the regioselective reduction of 4,6-O-benzylidene acetal groups.

Recently, we reported the synthesis of a new fluorous benzylidene acetal group for the protection of 1,3-diol compounds [15]. Efficient and expeditious syntheses of natural products [16], oligosaccharides [15], 
and modified monosaccharides have been accomplished by utilizing regioselective ring-opening reduction of fluorous benzylidene acetal groups and solid-phase extraction with a fluorous reversephase silica gel column. In the course of our studies on the expeditious synthesis of these products using fluorous benzylidene acetal groups, we isolated an interesting side product, the acyclic compound 3, during the regioselective ring-opening reduction of methyl 2,3-di-O-benzyl-4,6-OF benzylidene- $\alpha$-D-allopyranoside 1 with $\mathrm{PhBCl}_{2} / \mathrm{Et}_{3} \mathrm{SiH}$ (Scheme 1, Eq. 1). This unexpected side reaction is caused by the reductive cleavage of the fluorous benzylidene acetal group and subsequent endocyclic cleavage of the pyranosides. When methyl 2,3-di- $O$-benzyl-4,6- $O$ - ${ }^{\mathrm{F}}$ benzylidene- $\alpha$-Dglucopyranoside 4 and phenyl 2,3-di- $O$-benzyl-4,6- $O-{ }^{\mathrm{F}}$ benzylidene $\beta$-D-allopyranoside 6 were reacted under the same reaction conditions, this unexpected side reaction was not observed (Scheme 1, Eqs. 2,3).

Only a few reports have been published so far on the anomerization [17-24] and attachment of nucleophiles at $\mathrm{C} 1$ [25-32] via the endocyclic cleavage of glycosides. To the best of our knowledge, the side reaction described here is the first example of the endocyclic cleavage of methyl $\alpha$-D-allopyranoside derivatives with $\mathrm{PhBCl}_{2} / \mathrm{Et}_{3} \mathrm{SiH}$. Here, we provide detailed results of the ring cleavage reaction of hexopyranosides bearing axial substituents at $\mathrm{C} 1$ and $\mathrm{C} 3$.

Scheme 1. Reductive cleavage of fluorous benzylidene acetal group using $\mathrm{PhBCl}_{2} / \mathrm{Et}_{3} \mathrm{SiH}$.
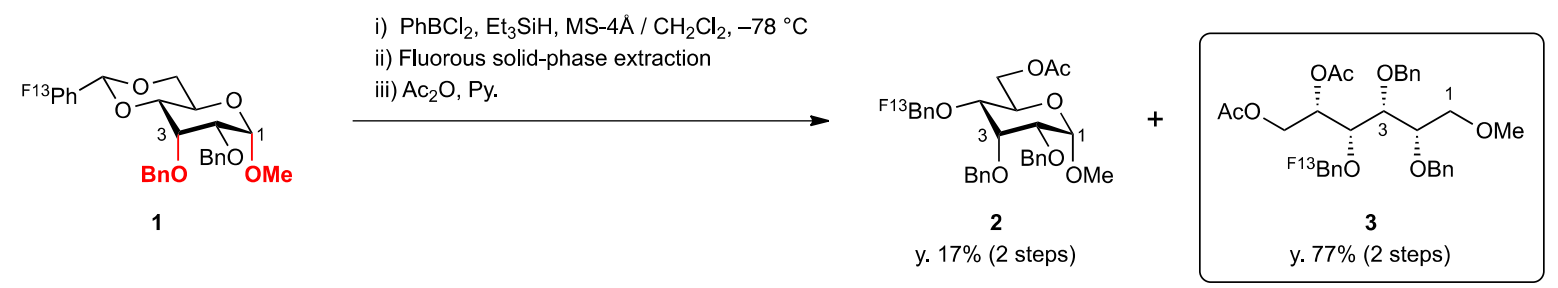

[Eq. 1]

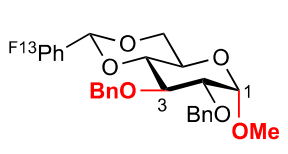

i) $\mathrm{PhBCl}_{2}, \mathrm{Et}_{3} \mathrm{SiH}, \mathrm{MS}-4 \AA / \mathrm{CH}_{2} \mathrm{Cl}_{2},-78^{\circ} \mathrm{C}$

ii) Fluorous solid-phase extraction

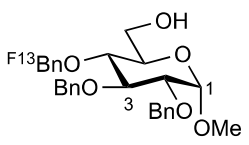

[Eq. 2]

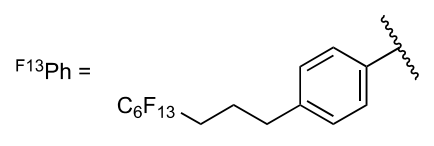

4

5: y. $96 \%$

i) $\mathrm{PhBCl} 2, \mathrm{Et} 3 \mathrm{SiH}, \mathrm{MS}-4 \AA / \mathrm{CH}_{2} \mathrm{Cl}_{2},-78^{\circ} \mathrm{C}$

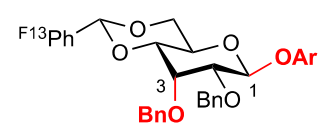

ii) Fluorous solid-phase extraction

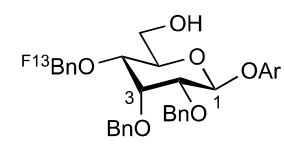

${ }^{\mathrm{F} 13} \mathrm{Bn}=$

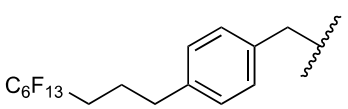

[Eq. 3]

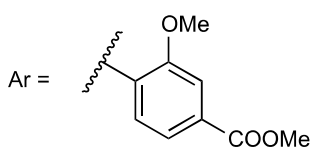

\section{Results and Discussion}

Initially, methyl 2,3-di-O-benzyl-4,6- $O$-benzylidene- $\alpha$-D-allopyranoside $\mathbf{8}$ was reacted with $\mathrm{PhBCl}_{2}$ (5.0 equiv.) and $\mathrm{Et}_{3} \mathrm{SiH}$ (4.5 equiv.) in $\mathrm{CH}_{2} \mathrm{Cl}_{2}$ at $-78{ }^{\circ} \mathrm{C}$. The purification of the crude product by silica gel column chromatography unexpectedly gave an acyclic derivative bearing a boronate ester as the main product [33]. Thus, to remove the phenylboronate group from the acyclic alditol derivative, an octadecyl silica gel (ODS) column was used instead of a fluorous reverse-phase silica gel column. The crude product was loaded onto the ODS column, after which the column was eluted successively with $40 \%$ aq. $\mathrm{MeOH}$ and then with $\mathrm{MeOH}$. The methanol fraction subsequently was evaporated, and the residue was treated with $\mathrm{Ac}_{2} \mathrm{O}$ and pyridine to give acyclic derivative 19 in $78 \%$ yield. In the case of methyl $\beta$-D-allopyranoside 11, the acyclic derivative 19 and 4-O-benzyl derivative 23 were obtained 
in $17 \%$ and $46 \%$ yields, respectively. However, the endocyclic cleavage of methyl $\alpha$-D-glucopyranoside 12 and methyl $\alpha$-D-galactopyranoside 13 was not observed. These results suggest that the hexopyranoside bearing axial substituents at $\mathrm{C} 1$ and $\mathrm{C} 3$ preferentially undergo endocyclic cleavage. To test the generality of this new finding, we examined the ring opening of various hexopyranosides bearing axial substituents at $\mathrm{C} 1$ and $\mathrm{C} 3$ under the same reaction conditions. The results are summarized in Table 1. When the reactions were carried out using methyl $\alpha$-D-allopyranoside derivatives $\mathbf{9}$ and $\mathbf{1 0}$ bearing methoxymethyl ethers and benzoyl esters at $\mathrm{C} 2$ and $\mathrm{C} 3$, the number of spots observed by thin-layer chromatography (TLC) was so large that the spots could not be identified. In the cases of methyl $\alpha$-Dgulopyranoside 14, allyl $\alpha$-D-allopyranoside 15, and methyl $\alpha$-D-ribo-hexopyranoside 16, the reactions proceeded smoothly to give the desired acyclic compounds 26, 27, and 28 in high yields. Additionally, the reaction involving hexopyranosides $\mathbf{1 7}$ and $\mathbf{1 8}$ bearing an axial substituent at $\mathrm{C} 2$ gave the acyclic compound 29 and the 4-O-benzylated compound $\mathbf{3 0}$ in $27 \%$ and $83 \%$ yields, respectively.

Table 1. Synthesis of acyclic derivatives from alkyl 4,6- $O$-benzylidene- $\alpha$-D-hexopyranosides.
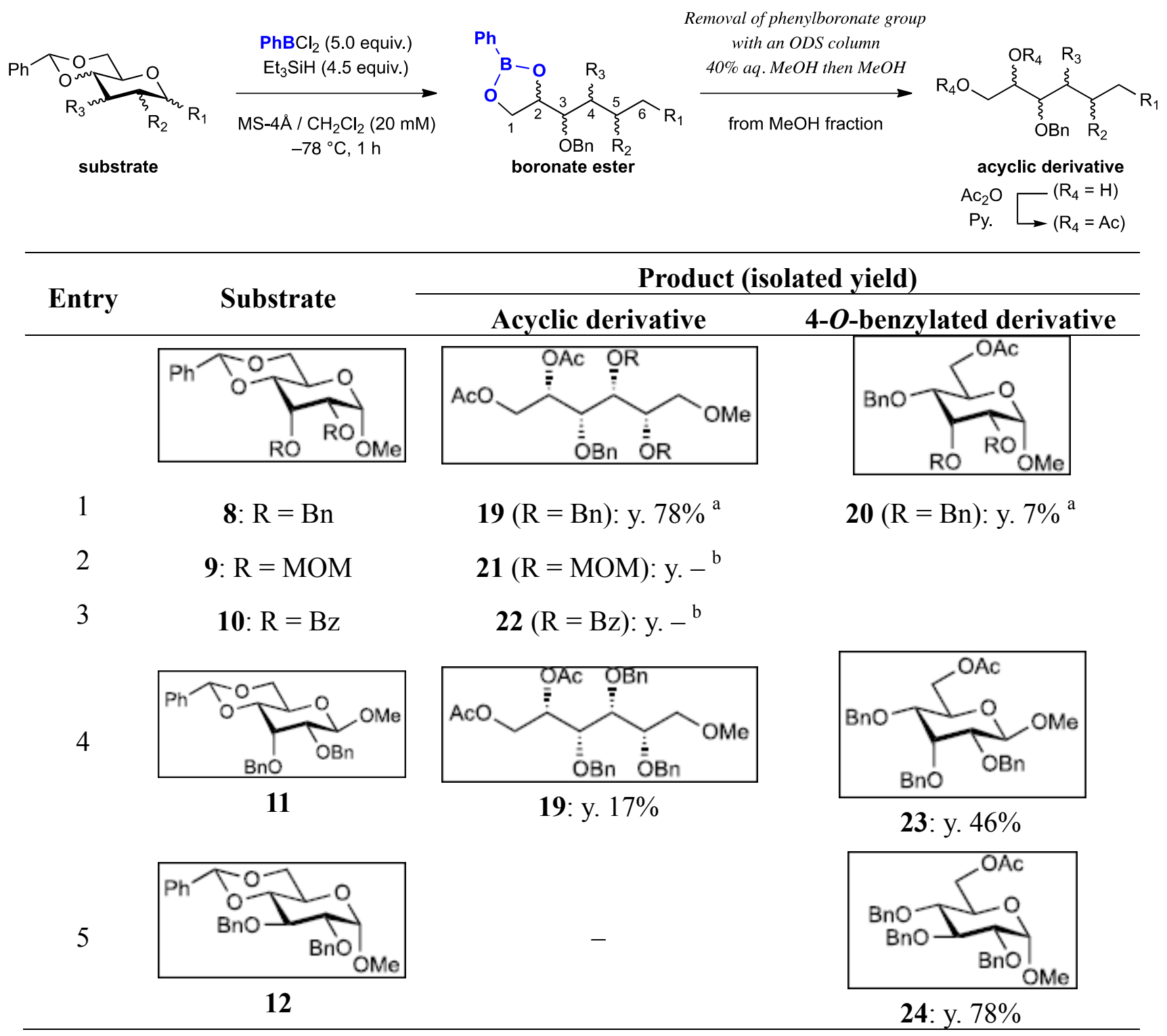
Table 1. Cont.

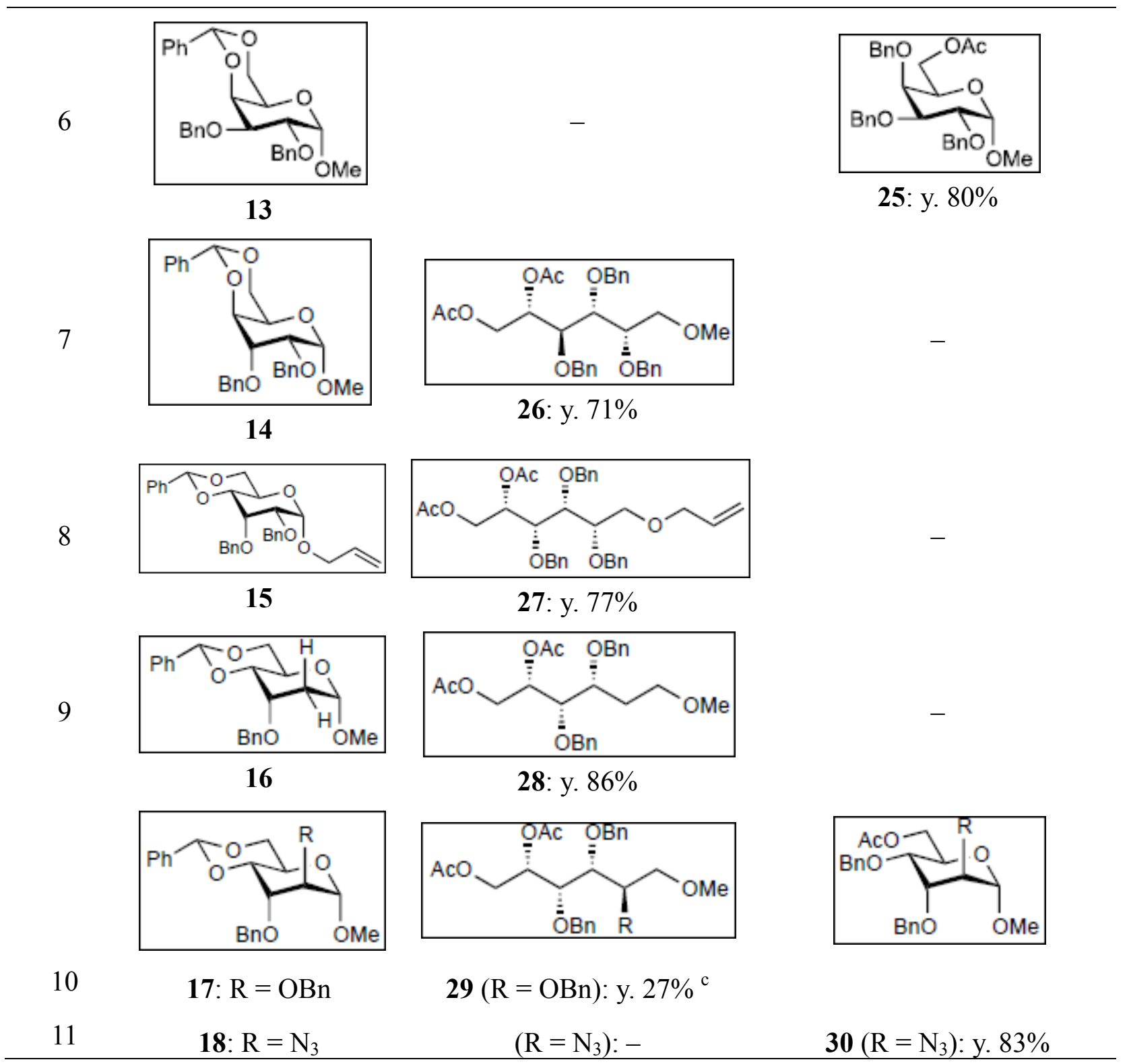

${ }^{\mathrm{a}}$ When 3.4 equiv. of $\mathrm{PhBCl}_{2}$ and 3.0 equiv. of $\mathrm{Et}_{3} \mathrm{SiH}$ were used, acyclic compound 19 and 4-Obenzylated compound $\mathbf{2 0}$ were obtained in 59\% and 19\% yields, respectively; ${ }^{\mathrm{b}}$ Many spots were observed by TLC; ${ }^{\mathrm{c}}$ An inseparable mixture was obtained as a main product.

We expected the hydroxyl group at C6 of the hexopyranosides to play an important role in cleavage of the bond between the anomeric carbon $\mathrm{C} 1$ and the pyranose ring oxygen atom $\mathrm{O} 5$ during endocyclic cleavage of hexopyranosides bearing axial substituents at $\mathrm{C} 1$ and $\mathrm{C} 3$ with $\mathrm{PhBCl}_{2} / \mathrm{Et}_{3} \mathrm{SiH}$ because a 1,2-boronate ester derivative was isolated as an intermediate. Therefore, methyl 2,3,4,6-tetra- $O$-benzyl$\alpha$-D-allopyranoside 31 and methyl 2,3,4-tri- $O$-benzyl- $\alpha$-D-allopyranoside 32 were reacted with $\mathrm{PhBCl}_{2}$ and $\mathrm{Et}_{3} \mathrm{SiH}$. As shown in Table 2, compound 31 gave methyl 3,4,6-tri- $O$-benzyl- $\alpha$-D-allopyranoside 33 and the starting material 31 in $28 \%$ and $52 \%$ yields, respectively. Although the reaction of 6-hydroxyderivative 32 at $-78{ }^{\circ} \mathrm{C}$ gave the desired acyclic derivative 34 with only a $10 \%$ yield, the yield reached $92 \%$ when the reaction was carried out at $-19^{\circ} \mathrm{C}$. 
On the basis of these experimental data, the pathway for $\mathrm{PhBCl}_{2}$-induced endocyclic cleavage of hexopyranosides with 1,3-diaxial substituents is speculated to be that shown in Scheme 2. The endocyclic cleavage is initiated by bond formation between the boron atom and oxygen atom O6 followed by chelation of the boron atom at ring oxygen atom O5. This interaction promotes cleavage of the endocyclic $\mathrm{C} 1-\mathrm{O} 5$ bond and formation of acyclic oxacarbenium ion $\mathbf{V}$. Before or after rotation around the $\mathrm{C} 1-\mathrm{C} 2$ bond, the addition of chloride ion from $\mathrm{PhBCl}_{2}$ to cation $\mathbf{V}$ followed by nucleophilic substitution with hydride ion (Path A) or direct addition of hydride ion to cation $\mathbf{V}$ (Path $\mathrm{B}$ ) gives boronate ester VII. The major factor in the endocyclic cleavage of methyl $\alpha$-D-allopyranoside $\mathbf{8}$ is due to steric strain of pyranosidic ring caused by steric repulsions between the substituents at $\mathrm{C} 1$ and C-3. Hexopyranosides $\mathbf{1 2}$ and $\mathbf{1 3}$ in which the pyranosidic rings are stabilized by the equatorial substituent at C-3 do not produce the corresponding acyclic derivatives. In the case of the reaction of hexopyranoside 11, the equatorial methoxy group at $\mathrm{C}-1$ sterically hinders bond formation between the boron atom and O5/O6 to give alditol derivative 19 in low yield. Since the ${ }^{4} \mathrm{C}_{1}$ conformation of altropyranoside $\mathbf{1 7}$ or $\mathbf{1 8}$ bearing axial substituents at $\mathrm{C} 1, \mathrm{C} 2$, and $\mathrm{C} 3$ is rapidly converted into the more stable ${ }^{1} \mathrm{C}_{4}$ conformation in which all the substituents are equatorial after the benzylidene acetal group is cleaved, the altropyranosides give alditol derivative 29 in low yield and 4-O-benzylated compound 30 in high yield. The endocyclic cleavage of 6-hydroxy-derivative 32 at $-78{ }^{\circ} \mathrm{C}$ results in the lower yield because the formation of $\mathbf{I V}$ is inhibited at the lower temperature, although the reaction from III to IV proceeds smoothly at the higher temperature.

However, the above-mentioned mechanism is highly speculative because of the lack of enough experimental data for supporting it. Therefore, we are now making efforts to get essential data for clarifying the mechanism by several experiments. We will report the results in the near future.

Table 2. Synthesis of acyclic derivatives from methyl 2,3,4,6-tetra- $O$-benzyl- $\alpha$-Dallopyranoside and 2,3,4-tri-O-benzyl- $\alpha$-D-allopyranoside.

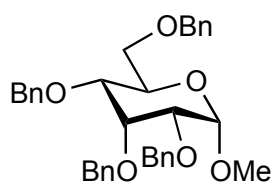

31

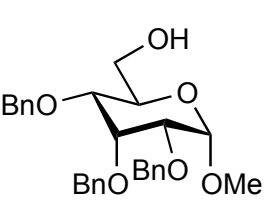

32

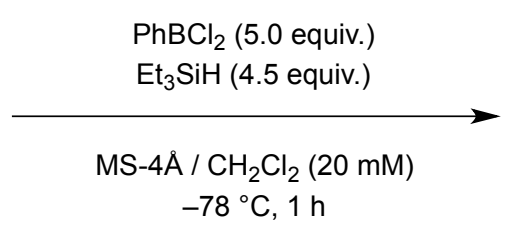

$-78{ }^{\circ} \mathrm{C}, 1 \mathrm{~h}$

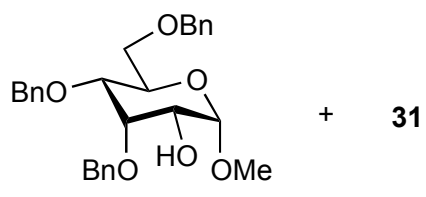

33: y. $28 \%$ y. $52 \%$

\begin{tabular}{llll}
\hline Entry & Reaction temperature $\left({ }^{\circ} \mathbf{C}\right)$ & $\begin{array}{l}\text { Yield of acyclic } \\
\text { compound 34 }(\mathbf{\%})\end{array}$ & $\begin{array}{l}\text { Yield of recovered } \\
\text { starting material 32 } \mathbf{( \% )}\end{array}$ \\
\hline 1 & -78 & 10 & 80 \\
2 & -60 & 53 & 41 \\
3 & -50 & 70 & 24 \\
4 & -40 & 80 & 18 \\
5 & -19 & 92 & - \\
\hline
\end{tabular}


Scheme 2. Proposed reaction mechanism.
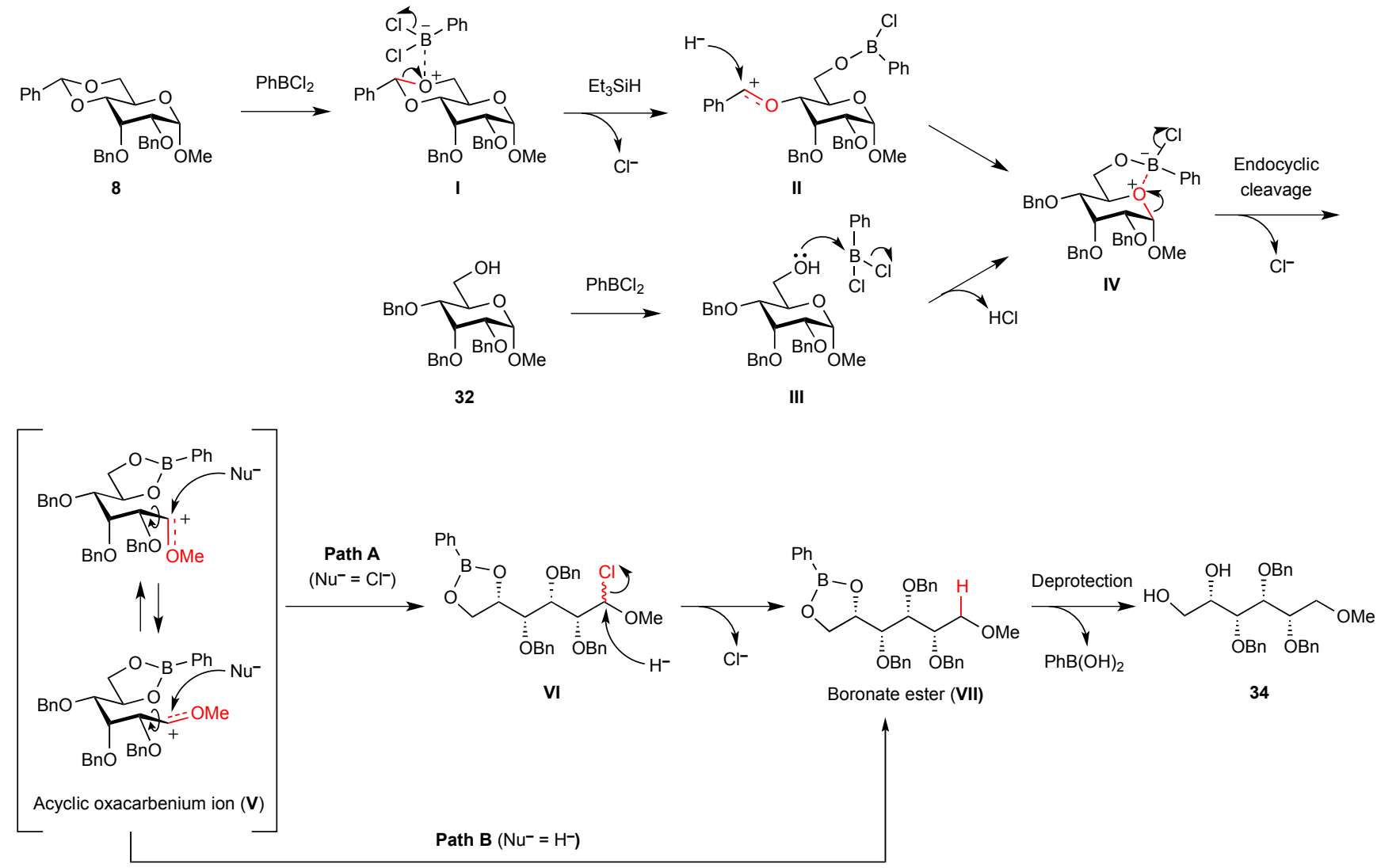

\section{Experimental}

\subsection{General}

${ }^{1} \mathrm{H}$ - and ${ }^{13} \mathrm{C}$-NMR spectra were measured using a Bruker Avance DPX-250 spectrometer. $J$ values were recorded in Hertz, and the abbreviations used were s (singlet), d (doublet), $\mathrm{t}$ (triplet), $\mathrm{m}$ (multiplet), and br (broad). Chemical shifts are expressed in $\delta$ values relative to the internal standard TMS. Octadecyl silica gel column chromatography was carried out using COSMOSIL $75 \mathrm{C}_{18}$-OPN ( $75 \mu \mathrm{m}$, Nacalai Tesque) column. TLC was carried out on Merck silica gel $60 \mathrm{~F} 254$ plates. $\mathrm{PhBCl}_{2}$ and $\mathrm{Et}_{3} \mathrm{SiH}$ were obtained from Sigma-Aldrich and Acros Organics, respectively.

\subsection{General Procedure for Endocyclic Cleavage with $\mathrm{PhBCl}_{2}$ and $\mathrm{Et}_{3} \mathrm{SiH}$}

A suspension of methyl 2,3-di- $O$-benzyl-4,6- $O$-benzylidene- $\alpha$-D-allopyranoside 8 (50 mg, $0.108 \mathrm{mmol}$ ) and MS-4 $\AA$ (250 mg) in dry $\mathrm{CH}_{2} \mathrm{Cl}_{2}(5.4 \mathrm{~mL})$ was stirred for $1 \mathrm{~h}$ at room temperature under argon. Next, $\mathrm{Et}_{3} \mathrm{SiH}\left(77 \mu \mathrm{L}, 0.486 \mathrm{mmol}, 4.5\right.$ equiv.) was added to the suspension at $-78{ }^{\circ} \mathrm{C}$, after which a solution of $\mathrm{PhBCl}_{2}\left(70 \mu \mathrm{L}, 0.541 \mathrm{mmol}, 5.0\right.$ equiv.) in $\mathrm{CH}_{2} \mathrm{Cl}_{2}(1 \mathrm{~mL})$ was added over $1 \mathrm{~h}$ via a syringe pump. After stirring for $1 \mathrm{~h}$ at the same temperature, the reaction mixture was quenched with $\mathrm{Et}_{3} \mathrm{~N}$ $(0.5 \mathrm{~mL})$ and $\mathrm{MeOH}(0.5 \mathrm{~mL})$ and then filtered through Celite. The filtrate was subsequently washed with saturated $\mathrm{NaHCO}_{3}$ solution $(5 \mathrm{~mL})$ and brine $(5 \mathrm{~mL})$, dried over $\mathrm{Na}_{2} \mathrm{SO}_{4}$, filtered, and concentrated. The residue was then loaded onto an octadecyl silica gel column, which was eluted successively with $40 \%$ aq. $\mathrm{MeOH}$ and $\mathrm{MeOH}$. Next, the $\mathrm{MeOH}$ fraction was concentrated to give the 
residue containing the acyclic diol. The residue was then redissolved in pyridine $(0.5 \mathrm{~mL})$, after which acetic anhydride $(0.5 \mathrm{~mL})$ was added. After stirring for $3 \mathrm{~h}$ at room temperature, the reaction mixture was poured into $\mathrm{MeOH}$ at $0{ }^{\circ} \mathrm{C}$ and stirred for $10 \mathrm{~min}$. The mixture was evaporated and co-evaporated with toluene. Finally, the residue was subjected to preparative thin-layer chromatography (hexane/EtOAc $=3: 2 \mathrm{v} / \mathrm{v}$ ) to give allitol derivative $19(46.7 \mathrm{mg}, 78 \%$ yield).

(2S,3R,4R,5S)-1,2-Bis(acetoxy)-3,4,5-tris(benzyloxy)-6-methoxyhexane (19). Colorless syrup; $R_{\mathrm{f}}=0.59$ (hexane/EtOAc $=3: 2 \mathrm{v} / \mathrm{v})$; IR $\left(\mathrm{NaCl}\right.$, neat): $1745 \mathrm{~cm}^{-1} ;{ }^{1} \mathrm{H}-\mathrm{NMR}\left(250 \mathrm{MHz}, \mathrm{CDCl}_{3}\right): \delta 7.33-7.25$ $(15 \mathrm{H}, \mathrm{m}, \mathrm{Ar} H), 5.48\left(1 \mathrm{H}, \mathrm{ddd}, J_{2,3}=4.0 \mathrm{~Hz}, J_{2,1}=2.7 \mathrm{~Hz}, J_{2,1^{\prime}}=7.3 \mathrm{~Hz}, \mathrm{H}-2\right), 4.73,4.68(2 \mathrm{H}$, each d, $\left.J=11.3 \mathrm{~Hz}, \mathrm{PhCH}_{2}\right), 4.71,4.58\left(2 \mathrm{H}\right.$, each d, $\left.J=11.6 \mathrm{~Hz}, \mathrm{PhCH}_{2}\right), 4.60\left(2 \mathrm{H}, \mathrm{s}, \mathrm{PhCH}_{2}\right), 4.43(1 \mathrm{H}, \mathrm{dd}$, $\left.J_{1,2}=2.7 \mathrm{~Hz}, J_{1,1^{\prime}}=12.2 \mathrm{~Hz}, \mathrm{H}-1\right), 4.24\left(1 \mathrm{H}, \mathrm{dd}, J_{1^{\prime}, 2}=7.3 \mathrm{~Hz}, J_{1,1^{\prime}}=12.2 \mathrm{~Hz}, \mathrm{H}-1^{\prime}\right), 3.93(1 \mathrm{H}, \mathrm{dd}$ t-like, $\left.J_{3,2}=4.0 \mathrm{~Hz}, J_{3,4}=4.4 \mathrm{~Hz}, \mathrm{H}-3\right), 3.84(2 \mathrm{H}, \mathrm{m}, \mathrm{H}-4,5), 3.61\left(1 \mathrm{H}, \mathrm{dd}, J_{6,5}=3.0 \mathrm{~Hz}, J_{6,6^{\prime}}=10.4 \mathrm{~Hz}\right.$, H-6), $3.54\left(1 \mathrm{H}, \mathrm{dd}, J_{6^{\prime}, 5}=4.7 \mathrm{~Hz}, J_{6^{\prime}, 6}=10.4 \mathrm{~Hz}, \mathrm{H}-6^{\prime}\right), 3.30\left(3 \mathrm{H}, \mathrm{s}, \mathrm{OCH}_{3}\right), 1.99,1.97(6 \mathrm{H}$, each s, $\left.\mathrm{CH}_{3} \times 2\right) ;{ }^{13} \mathrm{C}-\mathrm{NMR}\left(63 \mathrm{MHz}, \mathrm{CDCl}_{3}\right): \delta 170.7,169.8,138.4,137.9,137.7,128.3,128.24,128.22$, $128.17,128.08,128.03,127.97,127.7,127.63,127.57,127.4,78.4,78.2,78.1,73.8,72.8,72.3,71.85$, $71.79,63.3,58.9,21.0,20.7$.

(2S,3S,4R,5S)-1,2-Bis(acetoxy)-3,4,5-tris(benzyloxy)-6-methoxyhexane (26). Colorless syrup; $R_{\mathrm{f}}=0.63$ (hexane/EtOAc $=3: 2 \mathrm{v} / \mathrm{v})$; IR $\left(\mathrm{NaCl}\right.$, neat): $1744 \mathrm{~cm}^{-1} ;{ }^{1} \mathrm{H}-\mathrm{NMR}\left(250 \mathrm{MHz}, \mathrm{CDCl}_{3}\right): \delta 7.39-7.20$ $(15 \mathrm{H}, \mathrm{m}, \operatorname{Ar} H), 5.38\left(1 \mathrm{H}, \mathrm{ddd}, J_{2,3}=5.1 \mathrm{~Hz}, J_{2,1}=3.6 \mathrm{~Hz}, J_{2,1^{\prime}}=7.1 \mathrm{~Hz}, \mathrm{H}-2\right), 4.77(2 \mathrm{H}$, each d, $\left.J=11.4 \mathrm{~Hz}, \mathrm{PhCH}_{2}\right), 4.66,4.44\left(2 \mathrm{H}\right.$, each s, $\left.J=11.8 \mathrm{~Hz}, \mathrm{PhCH}_{2}\right), 4.64\left(2 \mathrm{H}, \mathrm{s}, \mathrm{PhCH}_{2}\right), 4.30(1 \mathrm{H}, \mathrm{dd}$, $\left.J_{1,2}=3.6 \mathrm{~Hz}, J_{1,1^{\prime}}=12.0 \mathrm{~Hz}, \mathrm{H}-1\right), 4.05\left(1 \mathrm{H}, \mathrm{dd}, J_{1^{\prime}, 2}=7.1 \mathrm{~Hz}, J_{1^{\prime}, 1}=12.0 \mathrm{~Hz}, \mathrm{H}-1\right), 3.92-3.77(3 \mathrm{H}, \mathrm{m}$, $\mathrm{H}-3,4,5), 3.74\left(1 \mathrm{H}, \mathrm{dd}, J_{6,5}=3.6 \mathrm{~Hz}, J_{6,6^{\prime}}=10.1 \mathrm{~Hz}, \mathrm{H}-6\right), 3.59\left(1 \mathrm{H}, \mathrm{dd}, J_{6^{\prime}, 5}=4.0 \mathrm{~Hz}, J_{6^{\prime}, 6}=10.1 \mathrm{~Hz}\right.$, $\left.\mathrm{H}^{-1}{ }^{\prime}\right), 3.35\left(3 \mathrm{H}, \mathrm{s}, \mathrm{OCH}_{3}\right), 2.01,1.97\left(6 \mathrm{H}\right.$, each s, $\left.\mathrm{CH}_{3} \times 2\right) ;{ }^{13} \mathrm{C}-\mathrm{NMR}\left(63 \mathrm{MHz}, \mathrm{CDCl}_{3}\right): \delta 170.5$, 170.2 , 138.4, 138.3, 138.0, 128.31, 128.29, 128.0, 127.9, 127.7, 127.60, 127.58, 127.52, 78.5, 78.3, 77.0 (overlapped with $\mathrm{CDCl}_{3}$ ), 74.5, 73.9, 72.0, 71.3, 71.2, 63.0, 58.9, 20.9, 20.7.

(2S,3R,4R,5S)-6-(Allyloxy)-1,2-bis(acetoxy)-3,4,5-tris(benzyloxy)hexane (27). Colorless syrup; $R_{\mathrm{f}}=0.50$ (hexane/EtOAc $=3: 2 \mathrm{v} / \mathrm{v})$; IR ( NaCl, neat): $1744 \mathrm{~cm}^{-1} ;{ }^{1} \mathrm{H}$ NMR $\left(250 \mathrm{MHz}, \mathrm{CDCl}_{3}\right): \delta 7.34-7.24$ $(15 \mathrm{H}, \mathrm{m}, \mathrm{Ar} H), 5.87\left(1 \mathrm{H}, \mathrm{ddt}, J=5.5 \mathrm{~Hz}, J=10.4 \mathrm{~Hz}, J=17.2 \mathrm{~Hz}, \mathrm{CH}_{2} \mathrm{CH}=\mathrm{CH}_{2}\right), 5.49(1 \mathrm{H}$, ddd, $\left.J_{2,3}=3.8 \mathrm{~Hz}, J_{2,1}=2.7 \mathrm{~Hz}, J_{2,1^{\prime}}=7.3 \mathrm{~Hz}, \mathrm{H}-2\right), 5.23\left(1 \mathrm{H}, \mathrm{dq}, J=1.6 \mathrm{~Hz}, J=17.2 \mathrm{~Hz}, \mathrm{CH}_{2} \mathrm{CH}=\mathrm{CH}_{2}\right)$, $5.14\left(1 \mathrm{H}, \mathrm{dq}, J=1.3 \mathrm{~Hz}, J=10.4 \mathrm{~Hz}, \mathrm{CH}_{2} \mathrm{CH}=\mathrm{CH}_{2}\right), 4.72,4.60\left(2 \mathrm{H}\right.$, each d, $\left.J=11.7 \mathrm{~Hz}, \mathrm{PhCH}_{2}\right)$, 4.70, $4.60\left(4 \mathrm{H}\right.$, each s, $\left.\mathrm{PhCH}_{2} \times 2\right), 4.42\left(1 \mathrm{H}, \mathrm{dd}, J_{1,2}=2.7 \mathrm{~Hz}, J_{1,1^{\prime}}=12.2 \mathrm{~Hz}, \mathrm{H}-1\right), 4.24(1 \mathrm{H}, \mathrm{dd}$, $\left.J_{1^{\prime}, 2}=7.3 \mathrm{~Hz}, J_{1^{\prime}, 1}=12.2 \mathrm{~Hz}, \mathrm{H}-1^{\prime}\right), 3.95-3.82\left(5 \mathrm{H}, \mathrm{m}, \mathrm{H}-3,4,5, \mathrm{CH}_{2} \mathrm{CH}=\mathrm{CH}_{2}\right), 3.68(1 \mathrm{H}, \mathrm{dd}$, $\left.J_{6,5}=3.0 \mathrm{~Hz}, J_{6,6^{\prime}}=10.4 \mathrm{~Hz}, \mathrm{H}-6\right), 3.59\left(1 \mathrm{H}, \mathrm{dd}, J_{6^{\prime}, 5}=5.2 \mathrm{~Hz}, J_{6^{\prime}, 6}=10.4 \mathrm{~Hz}, \mathrm{H}-6\right), 1.99,1.97(6 \mathrm{H}$, each s, $\left.\mathrm{CH}_{3} \times 2\right) ;{ }^{13} \mathrm{C}-\mathrm{NMR}\left(63 \mathrm{MHz}, \mathrm{CDCl}_{3}\right): \delta 170.7,169.8,138.6,138.0,137.8,134.8,128.29$, $128.26,128.21,128.12,128.05,127.8,127.7,127.6,127.4,116.7,78.6,78.4,78.3,73.8,72.9,72.5$, $72.2,71.9,69.7,63.3,21.0,20.8$.

(2S,3S,4R)-1,2-Bis(acetoxy)-3,4-bis(benzyloxy)-6-methoxyhexane (28). Colorless syrup; $R_{\mathrm{f}}=0.55$ (hexane/EtOAc $=1: 1 \mathrm{v} / \mathrm{v})$; IR $\left(\mathrm{NaCl}\right.$, neat): $1745 \mathrm{~cm}^{-1} ;{ }^{1} \mathrm{H}-\mathrm{NMR}\left(250 \mathrm{MHz}, \mathrm{CDCl}_{3}\right): \delta 7.38-7.25$ $(10 \mathrm{H}, \mathrm{m}, \mathrm{Ar} H), 5.28\left(1 \mathrm{H}, \mathrm{ddd}, J_{2,3}=4.8 \mathrm{~Hz}, J_{2,1}=2.6 \mathrm{~Hz}, J_{2,1^{\prime}}=6.9 \mathrm{~Hz}, \mathrm{H}-2\right), 4.72,4.55(2 \mathrm{H}$, each d, $\left.J=11.5 \mathrm{~Hz}, \mathrm{PhCH}_{2}\right), 4.68,4.63\left(2 \mathrm{H}\right.$, each d, $\left.J=10.5 \mathrm{~Hz}, \mathrm{PhCH}_{2}\right), 4.48\left(1 \mathrm{H}, \mathrm{dd}, J_{1,2}=2.6 \mathrm{~Hz}\right.$, 
$\left.J_{1,1^{\prime}}=12.2 \mathrm{~Hz}, \mathrm{H}-1\right), 4.25\left(1 \mathrm{H}, \mathrm{dd}, J_{1^{\prime}, 2}=6.9 \mathrm{~Hz}, J_{1^{\prime}, 1}=12.2 \mathrm{~Hz}, \mathrm{H}-1^{\prime}\right), 3.80-3.73(2 \mathrm{H}, \mathrm{m}, \mathrm{H}-3,4)$, 3.54-3.35 (2H, m, H-6, 6'), $3.26\left(3 \mathrm{H}, \mathrm{s}, \mathrm{OCH}_{3}\right), 2.04,2.01\left(6 \mathrm{H}\right.$, each s, $\left.\mathrm{CH}_{3} \times 2\right), 1.92-1.84(2 \mathrm{H}, \mathrm{m}$, $\mathrm{H}-5) ;{ }^{13} \mathrm{C}-\mathrm{NMR}\left(63 \mathrm{MHz}, \mathrm{CDCl}_{3}\right): \delta 170.6,169.9,138.2,137.9,128.3,128.0,127.7,127.6,79.3,75.9$, $73.3,72.5,71.4,68.8,63.2,58.4,30.6,20.9,20.7$.

(2S,3R,4R,5R)-1,2-Bis(acetoxy)-3,4,5-tris(benzyloxy)-6-methoxyhexane (29). Colorless syrup; $R_{\mathrm{f}}=0.46$ (hexane/EtOAc $=3: 2 \mathrm{v} / \mathrm{v})$; IR $\left(\mathrm{NaCl}\right.$, neat): $1744 \mathrm{~cm}^{-1} ;{ }^{1} \mathrm{H}-\mathrm{NMR}\left(250 \mathrm{MHz}, \mathrm{CDCl}_{3}\right): \delta 7.67-7.20$ $(15 \mathrm{H}, \mathrm{m}, \mathrm{Ar} H), 5.40\left(1 \mathrm{H}, \mathrm{ddd}, J_{2,3}=3.4 \mathrm{~Hz}, J_{2,1}=2.8 \mathrm{~Hz}, J_{2,1^{\prime}}=7.3 \mathrm{~Hz}, \mathrm{H}-2\right), 4.79,4.70$ (2H, each d, $\left.J=11.3 \mathrm{~Hz}, \mathrm{PhCH}_{2}\right), 4.66,4.58\left(2 \mathrm{H}\right.$, each d, $\left.J=11.7 \mathrm{~Hz}, \mathrm{PhCH}_{2}\right), 4.62,4.51(2 \mathrm{H}$, each d, $J=11.6 \mathrm{~Hz}$, $\left.\mathrm{PhCH}_{2}\right), 4.54\left(1 \mathrm{H}, \mathrm{dd}, J_{1,2}=2.8 \mathrm{~Hz}, J_{1,1^{\prime}}=12.2 \mathrm{~Hz}, \mathrm{H}-1\right), 4.27\left(1 \mathrm{H}, \mathrm{dd}, J_{1^{\prime}, 2}=7.3 \mathrm{~Hz}, J_{1^{\prime}, 1}=12.2 \mathrm{~Hz}\right.$, H-1'), 3.93-3.85 (2H, m, H-3, 4), $3.79\left(1 \mathrm{H}\right.$, ddd q-like, $\left.J_{5,4}=4.8 \mathrm{~Hz}, J_{5,6}=4.8 \mathrm{~Hz}, J_{5,6^{\prime}}=4.8 \mathrm{~Hz}, \mathrm{H}-5\right)$, $3.57\left(1 \mathrm{H}, \mathrm{dd}, J_{6,5}=4.7 \mathrm{~Hz}, J_{6,6^{\prime}}=10.2 \mathrm{~Hz}, \mathrm{H}-6\right), 3.51\left(1 \mathrm{H}, \mathrm{dd}, J_{6^{\prime}, 5}=4.8 \mathrm{~Hz}, J_{6^{\prime}, 6}=10.2 \mathrm{~Hz}, \mathrm{H}-6^{\prime}\right), 3.30$ $\left(3 \mathrm{H}, \mathrm{s}, \mathrm{OCH}_{3}\right), 1.98\left(6 \mathrm{H}, \mathrm{s}, \mathrm{CH}_{3} \times 2\right) ;{ }^{13} \mathrm{C}-\mathrm{NMR}\left(63 \mathrm{MHz}, \mathrm{CDCl}_{3}\right): \delta 170.7,169.9,138.6,138.3,137.9$, $128.31,128.28,128.26,128.1,127.9,127.8,127.7,127.6,127.5,78.8,78.2,74.6,73.0,72.7,72.0$, $71.9,63.3,59.1,21.0,20.8$.

(2S,3R,4R,5S)-1,2-Dihydroxy-3,4,5-tris(benzyloxy)-6-methoxyhexane (34). Colorless syrup; $R_{\mathrm{f}}=0.24$ (hexane/EtOAc $=3: 2 \mathrm{v} / \mathrm{v})$; IR ( $\mathrm{NaCl}$, neat): $3444 \mathrm{~cm}^{-1} ;{ }^{1} \mathrm{H}-\mathrm{NMR}\left(250 \mathrm{MHz}, \mathrm{CDCl}_{3}\right): \delta 7.38-7.25$ $(15 \mathrm{H}, \mathrm{m}, \mathrm{ArH}), 4.73,4.61\left(2 \mathrm{H}\right.$, each d, $\left.J=11.6 \mathrm{~Hz}, \mathrm{PhCH}_{2}\right), 4.71\left(2 \mathrm{H}, \mathrm{s}, \mathrm{PhCH}_{2}\right), 4.68,4.55(2 \mathrm{H}$, each $\left.\mathrm{d}, J=11.4 \mathrm{~Hz}, \mathrm{PhCH}_{2}\right), 3.97\left(1 \mathrm{H}, 1 \mathrm{H}, \mathrm{dd}, J_{4,3}=3.5 \mathrm{~Hz}, J_{4,5}=6.1 \mathrm{~Hz}, \mathrm{H}-4\right), 3.90\left(1 \mathrm{H}, \mathrm{ddd}, J_{5,4}=6.1 \mathrm{~Hz}\right.$, $\left.J_{5,6}=3.5 \mathrm{~Hz}, J_{5,6^{\prime}}=4.6 \mathrm{~Hz}, \mathrm{H}-5\right), 3.93-3.84(1 \mathrm{H}, \mathrm{m}, \mathrm{H}-2$, overlapped with $\mathrm{H}-5), 3.76(1 \mathrm{H}$, dd, $\left.J_{3,4}=3.5 \mathrm{~Hz}, J_{3,2}=7.0 \mathrm{~Hz}, \mathrm{H}-3\right), 3.71-3.60\left(2 \mathrm{H}, \mathrm{m}, \mathrm{H}-1,1^{\prime}\right.$, overlapped with H-6, 6'), 3.66 (1H, dd, $\left.J_{6,5}=3.5 \mathrm{~Hz}, J_{6,6^{\prime}}=10.4 \mathrm{~Hz}, \mathrm{H}-6\right), 3.59\left(1 \mathrm{H}, \mathrm{dd}, J_{6^{\prime}, 5}=4.6 \mathrm{~Hz}, J_{6^{\prime}, 6}=10.4 \mathrm{~Hz}, \mathrm{H}-6^{\prime}\right), 3.35(3 \mathrm{H}, \mathrm{s}$, $\left.\mathrm{OCH}_{3}\right), 3.22(1 \mathrm{H}$, br d, $J=3.7 \mathrm{~Hz}, \mathrm{OH}), 2.17\left(1 \mathrm{H}\right.$, br s, OH); ${ }^{13} \mathrm{C}-\mathrm{NMR}\left(63 \mathrm{MHz}, \mathrm{CDCl}_{3}\right): \delta 138.02$, $137.95,137.90,128.43,128.41,128.08,128.06,128.01,127.9,127.8,79.4,79.3,78.1,73.9,73.2,72.7$, $71.81,71.78,63.9,59.2$.

\section{Conclusions}

The reaction of alkyl 4,6- $O$-benzylidene- $\alpha$-D-allopyranoside, 4,6- $O$-benzylidene- $\alpha$-D-gulopyranoside, and 4,6- $O$-benzylidene- $\alpha$-D-altropyranoside derivatives carrying 1,3-diaxial substituents with $\mathrm{PhBCl}_{2} / \mathrm{Et}_{3} \mathrm{SiH}$ gave 4-O-benzyl ethers and alditol derivatives formed by $\mathrm{C} 1 / \mathrm{O} 5$ bond cleavage. Because an acyclic boronate ester was isolated, the unexpected endocyclic cleavage is considered to proceed via an oxacarbenium ion intermediate produced by the chelation between O5/O6 of the pyranoside and $\mathrm{PhBCl}_{2}$ followed by nucleophilic substitution with a hydride ion at $\mathrm{C} 1$. The oxacarbenium ion could be employed as a valuable and versatile intermediate for stereoselective carbon-carbon, carbon-nitrogen, carbon-sulfur, and carbon-oxygen bond formations with a variety of nucleophiles. Further reactivity studies of this endocyclic cleavage are underway in our laboratory. The results of these studies will be reported in the near future. 


\section{Acknowledgements}

We wish to thank Ken-ichi Sato and Shoji Akai, Kanagawa University, for their helpful discussions. We also thank N. Higaki and Y. Nakagawa for technical assistance.

\section{References and Notes}

1. DeNinno, M.P.; Etienne, J.B.; Duplantier, K.C. A method for the selective reduction of carbohydrate 4,6-O-benzylidene acetals. Tetrahedron Lett. 1995, 36, 669-672.

2. Sakagami, M.; Hamana, H. A selective ring opening reaction of 4,6-O-benzylidene acetals in carbohydrates using trialkylsilane derivatives. Tetrahedron Lett. 2000, 41, 5547-5551.

3. Shie, C.-R.; Toone, Z.-H.; Kulkarni, S.S.; Uang, B.-J.; Hsu, C.-Y.; Hung, S.-C. Cu(OTf $)_{2}$ as an efficient and dual-purpose catalyst in the regioselective reductive ring opening of benzylidene acetals. Angew. Chem. Int. Ed. 2005, 44, 1665-1668.

4. Hernández-Torres, J.M.; Achkar, J.; Wei, A. Temperature-controlled regioselectivity in the reductive cleavage of $p$-methoxybenzylidene acetals. J. Org. Chem. 2004, 69, 7206-7211.

5. Sherman, A.A.; Mironov, Y.V.; Yudina, O.N.; Nifantiev, N.E. The presence of water improves reductive openings of benzylidene acetals with trimethylaminoborane and aluminium chloride. Carbohydr. Res. 2003, 338, 697-703.

6. Wang, C.-C.; Luo, S.-Y.; Shie, C.-R.; Hung, S.-C. Metal trifluoromethanesulfonate-catalyzed regioselective borane-reductive ring opening of benzylidene acetals: A concise synthesis of 1,4-dideoxy-1,4-imino-L-xylitol. Org. Lett. 2002, 4, 847-849.

7. Debenham, A.D.; Toone, E.J. Regioselective reduction of 4,6-O-benzylidenes using triethylsilane and $\mathrm{BF}_{3} \cdot \mathrm{Et}_{2} \mathrm{O}$. Tetrahedron:Asymmetry 2000, 11, 385-387.

8. Rao, K.V.; Patil, P.R.; Atmakuri, S.; Kartha, K.P.R. Iodide-sodium cyanoborohydride-mediated reductive ring opening of 4,6-benzylidene acetals of hexopyranosides. Carbohydr. Res. 2010, 345, 2709-2713.

9. Panchadhayee, R.; Misra, A.K. Regioselective reductive ring opening of benzylidene acetals using triethylsilane and iodide. Synlett 2010, 8, 1193-1196.

10. Daragics, K.; Fügedi, P. Regio- and chemoselective reductive cleavage of 4,6- $O$-benzylidene-type acetals of hexopyranosides using $\mathrm{BH}_{3} \cdot \mathrm{THF}-\mathrm{TMSOTf}$. Tetrahedron Lett. 2009, 50, 2914-2916.

11. Saito, S.; Kuroda, A.; Tanaka, K.; Kimura, R. A novel reducing system for acetal cleavage: $\mathrm{BH}_{3} \cdot \mathrm{S}\left(\mathrm{CH}_{3}\right)_{2}-\mathrm{BF}_{3} \cdot \mathrm{O}\left(\mathrm{C}_{2} \mathrm{H}_{5}\right)_{2}$ combination. Synlett 1996, 3, 231-233.

12. Tani, S.; Sawadi, S.; Kojima, M.; Akai, S.; Sato, K. A novel method for regioselective ringopening reduction of 4,6-O-benzylidene hexopyranoside derivatives using $\mathrm{CoCl}_{2}$ and $\mathrm{BH}_{3} \cdot \mathrm{THF}_{\text {. }}$. Tetrahedron Lett. 2007, 48, 3103-3104.

13. Ohlin, M.; Johnsson, R; Ellervik, U. Regioselective reductive openings of 4,6-benzylidene acetals: synthetic and mechanistic aspects. Carbohydr. Res. 2011, 346, 1358-1370.

14. Denmark, S.E.; Almstead, N.G. Studies on the mechanism and origin of stereoselective opening of chiral dioxane acetals. J. Am. Chem. Soc. 1991, 113, 8089-8110.

15. Kojima, M.; Nakamura, Y; Takeuchi, S. A practical fluorous benzylidene acetal protecting group for a quick synthesis of disaccharides. Tetrahedron Lett. 2007, 48, 4431-4436. 
16. Kojima, M.; Nakamura, Y.; Ito, S.; Takeuchi, S. Total synthesis of macrocyclic glycosides, clemochinenosides A and B, and berchemolide, by fluorous mixture synthesis. Tetrahedron Lett. 2009, 50, 6143-6149.

17. Lidberg, B. Action of strong acids on acetylated glucosides. Acta Chem. Scand. 1949, 3, 1153-1169.

18. Morishima, N.; Koto, S.; Zen, S. A rapid anomerization of alkyl per-O-benzyl- $\beta$-Dglucopyranosides by titanium tetrachloride. Chem. Lett. 1979, 8, 749-750.

19. O’Brien, C.; Poláková, M.; Pitt, N.; Tosin, M.; Murphy, P.V. Glycosidation-anomerization reactions of 6,1-anhydroglucopyranuronic acid and anomerization of $\beta$-D-glucopyranosiduronic acids promoted by $\mathrm{SnCl}_{4}$. Chem. Eur. J. 2007, 13, 902-909.

20. Manabe, S.; Ishii, K.; Hashizume, D.; Koshino, H.; Ito, Y. Evidence for endocyclic cleavage of conformationally restricted glycopyranosides. Chem. Eur. J. 2009, 15, 6894-6901.

21. Satoh, H.; Hutter, J.; Lüthi, H.P.; Manabe, S.; Ishii, K.; Ito, Y. Low-barrier pathway for endocleavage induced anomerization of pyranosides with $\mathrm{N}$-benzyl-2,3-trans-oxazolidinone groups. Eur. J. Org. Chem. 2009, 1127-1131.

22. Manabe, S.; Ito, Y. Significant solvent effect in anomerization reaction of pyranosides with 2,3trans carbamate and carbonate. Tetrahedron Lett. 2009, 50, 4827-4829.

23. Pilgrim, W.; Murphy, P. $\mathrm{SnCl}_{4}{ }^{-}$and $\mathrm{TiCl}_{4}$-catalyzed anomerization of acylated $O-$ and $S$-glycosides: Analysis of factors that lead to higher $\alpha: \beta$ anomer ratios and reaction rates. J. Org. Chem. 2010, 75, 6747-6755.

24. Satoh, H.; Manabe, S.; Ito, Y.; Lüthi, H.P.; Laino, T.; Hutter, J. Endocyclic cleavage in glycosides with 2,3-trans cyclic protecting groups. J. Am. Chem. Soc. 2011, 133, 5610-5619.

25. Köster, R.; Penadés-Ullate, S.; Dahlhoff, W.V. Catalyzed acetal reduction with $>$ BH boranes $-1-O-$ alkyl(aryl)alditols, anhydroalditols, and 1-O-alditylalditols from $O$-glycopyranosides. Angew. Chem. Int. Ed. 1985, 24, 519-521.

26. Guindon, Y.; Anderson, P.C. Stereoelectronic effects in the ring cleavage of methyl glycopyranosides using dimethylboron bromide. Tetrahedron Lett. 1987, 28, 2485-2488.

27. Inghardt, T.; Frejd, T. Organoaluminum-induced opening of the pyranosidic ring of benzyl 2deoxy-2-C-methylpentopyranosides. J. Org. Chem. 1989, 54, 5539-5543.

28. Hashimoto, H.; Hayakawa, M. Synthesis of a new fucosidase inhibitor, 1,5-dideoxy-1,5-imino-Ltalitol, via cyanotrimethylsilanolysis of a $\beta$-D-ribofuranoside and its inhibitory activities. Chem. Lett. 1989, 18, 1881-1884.

29. Hashimoto, H.; Kawanishi, M.; Yuasa, H. New and facile synthetic routes to 5-thioaldohexopyranosides via aldose monothioacetal derivatives. Tetrahedron Lett. 1991, 32, 7087-7090.

30. Martin, O.R.; Rao, S.P.; Yang, T.-F.; Fotia, F. Chelation-controlled regio- and stereoselective $C$-allylation of glycosides and related cyclic hemiacetals. Synlett 1991, 702-704.

31. Olsson, R.; Rundström, P.; Frejd, T. Chelation-controlled regioselective endo cleavage and stereoselective C-1 alkylation of pentofuranosides. J. Chem. Soc. Perkin Trans. 1 1998, 785-790.

32. Olsson, R.; Berg, U.; Frejd, T. Endocyclic cleavage of glycosides. VI. Substituent effects of the alkylative endocyclic cleavage of glycosides Tetrahedron 1998, 54, 3935-3954. 
33. 1,2-Boronate ester: Colorless syrup; $R_{\mathrm{f}}=0.50$ (hexane/EtOAc $\left.=1: 1 \mathrm{v} / \mathrm{v}\right) ; \mathrm{IR}(\mathrm{NaCl}$, neat): 1602, 1441, 1397, 1327 $\mathrm{cm}^{-1} ;{ }^{1} \mathrm{H}-\mathrm{NMR}\left(250 \mathrm{MHz}, \mathrm{CDCl}_{3}\right): \delta 7.81-7.77\left(2 \mathrm{H}, \mathrm{m}, \mathrm{BC}_{6} H_{5}\right), 7.50-7.18$ $(18 \mathrm{H}, \mathrm{m}, \mathrm{Ar} H), 4.81\left(\mathrm{ddd}, J_{2,3}=3.7 \mathrm{~Hz}, J_{2,1}=7.3 \mathrm{~Hz}, J_{2,1^{\prime}}=8.3 \mathrm{~Hz}, \mathrm{H}-2\right), 4.72,4.57$ (2H, each d, $\left.J=11.7 \mathrm{~Hz}, \mathrm{PhCH}_{2}\right), 4.69,4.60\left(2 \mathrm{H}\right.$, each d, $\left.J=11.5 \mathrm{~Hz}, \mathrm{PhCH}_{2}\right), 4.68,4.63(2 \mathrm{H}$, each d, $J=11.4 \mathrm{~Hz}$, $\left.\mathrm{PhCH}_{2}\right), 4.51\left(1 \mathrm{H}, \mathrm{dd}, J_{1,2}=7.3 \mathrm{~Hz}, J_{1,1^{\prime}}=9.1 \mathrm{~Hz}, \mathrm{H}-1\right), 4.18\left(1 \mathrm{H}, \mathrm{dd}, J_{1^{\prime}, 2}=8.4 \mathrm{~Hz}, J_{1^{\prime}, 1}=9.0 \mathrm{~Hz}\right.$, H-1'), $4.08\left(1 \mathrm{H}\right.$, dd t-like, $\left.J_{3,4}=3.6 \mathrm{~Hz}, J_{3,2}=3.7 \mathrm{~Hz}, \mathrm{H}-3\right), 3.88\left(1 \mathrm{H}, \mathrm{dd}, J_{4,3}=3.6 \mathrm{~Hz}, J_{4,5}=6.2 \mathrm{~Hz}\right.$, $\mathrm{H}-4), 3.81\left(1 \mathrm{H}\right.$, ddd, $\left.J_{5,4}=6.2 \mathrm{~Hz}, J_{5,6}=3.3 \mathrm{~Hz}, J_{5,6^{\prime}}=4.5 \mathrm{~Hz}, \mathrm{H}-5\right), 3.66\left(1 \mathrm{H}, \mathrm{dd}, J_{6,5}=3.3 \mathrm{~Hz}\right.$, $\left.J_{6,6^{\prime}}=10.4 \mathrm{~Hz}, \mathrm{H}-1\right), 3.59\left(1 \mathrm{H}, \mathrm{dd}, J_{6^{\prime}, 5}=4.5 \mathrm{~Hz}, J_{6^{\prime}, 6}=10.4 \mathrm{~Hz}, \mathrm{H}-1^{\prime}\right), 3.35\left(3 \mathrm{H}, \mathrm{s}, \mathrm{OCH}_{3}\right)$; ${ }^{13} \mathrm{C}-\mathrm{NMR}\left(63 \mathrm{MHz}, \mathrm{CDCl}_{3}\right): \delta 138.2,138.1,138.0,134.9\left(\mathrm{BC}_{6} \mathrm{H}_{5}\right), 131.3\left(\mathrm{BC}_{6} \mathrm{H}_{5}\right), 128.4,128.3$, $128.24,128.17,128.0,127.9,127.8,127.7,127.61,127.57,80.2,78.2,78.0,77.3,74.0,73.5,72.4$, $71.8,67.5,59.1$. The structure of boronate ester was established by comparing ${ }^{1} \mathrm{H}-$ and ${ }^{13} \mathrm{C}-\mathrm{NMR}$ chemical shifts with those of similar compounds reported in the following literatures [34-37].

34. Crinch, D.; de la Mora, M.; Vinod, A.U. Influence of the 4,6-O-benzylidene, 4,6-Ophenylboronate, and 4,6-O-polystyrylboronate protecting groups on the stereochemical outcome of thioglycoside-based glycosylations mediated by 1-benzenesulfinyl piperidine/triflic anhydride and $N$-iodosuccinimide/trimethylsilyl triflate. J. Org. Chem. 2003, 68, 8142-8148.

35. Smith, J.M.; Borsenberger, V.; Raftery, J.; Sutherland, J.D. Exploratory studies to investigate a linked prebiotic origin of RNA and coded peptides. 2nd communication. Chem. Biodiv. 2004, 1, 1418-1451.

36. Bartoli, G.; Bosco, M.; Martino, E.D.; Marcantoni, E.; Sambri, L. Highly stereoselective and efficient addition of organocerium reagents to $s y n$ - $\beta$-alkyl- $\beta$-hydroxy- $\alpha$-methyl ketones by way of their titanium alkoxides-Synthesis of complex 1,3-diol units with three streodefined centres. Eur. J. Org. Chem. 2001, 15, 2901-2909.

37. Meiland, M.; Heinze, T.; Guenther, W.; Liebert, T. Seven-membered ring boronates at trans-diol moieties of carbohydrates. Tetrahedron Lett. 2009, 50, 469-472.

SampleAvailability: Samples of the compounds 8, 11-20 and 23-34 are available from the authors.

(C) 2011 by the authors; licensee MDPI, Basel, Switzerland. This article is an open access article distributed under the terms and conditions of the Creative Commons Attribution license (http://creativecommons.org/licenses/by/3.0/). 